\title{
Metallurgy Beyond Iron
}

\author{
Isabella Gallino ${ }^{\mathrm{A}, \mathrm{B}}$ and Ralf Busch ${ }^{\mathrm{A}}$ \\ A Saarland University, Chair for Metallic Materials, Campus C6.3, 66123 Saarbruecken, Germany \\ ${ }^{\mathrm{B}}$ Corresponding author. Email: i.gallino@mx.uni-saarland.de
}

Received 2008 December 17, accepted 2008 December 23

\begin{abstract}
Metallurgy is one of the oldest sciences. Its history can be traced back to $6000 \mathrm{BCE}$ with the discovery of Gold, and each new discovery - Copper, Silver, Lead, Tin, Iron and Mercury — marked the beginning of a new era of civilization. Currently there are 86 known metals, but until the end of the 17 th century, only 12 of these were known. Steel (Fe-C alloy) was discovered in the 11th century BCE; however, it took until $1709 \mathrm{CE}$ before we mastered the smelting of pig-iron by using coke instead of charcoal and started the industrial revolution. The metallurgy of nowadays is mainly about discovering better materials with superior properties to fulfil the increasing demand of the global market. Promising are the Glassy Metals or Bulk Metallic Glasses (BMGs) — discovered at first in the late 50s at the California Institute of Technology which are several times stronger than the best industrial steels and 10-times springier. The unusual structure that lacks crystalline grains makes BMGs so promising. They have a liquid-like structure that means they melt at lower temperatures, can be moulded nearly as easily as plastics, and can be shaped into features just $10 \mathrm{~nm}$ across. The best BMG formers are based on $\mathrm{Zr}, \mathrm{Pd}, \mathrm{Pt}, \mathrm{Ca}, \mathrm{Au}$ and, recently discovered, also Fe. They have typically three to five components with large atomic size mismatch and a composition close to a deep eutectic. Packing in such liquids is very dense, with a low content of free volume, resulting in viscosities that are several orders of magnitude higher than in pure metal melts.
\end{abstract}

Keyword: metallic glasses

\section{Introduction}

If we consider the abundance of elements on earth, the most abundant metallic elements are $\mathrm{Al}, \mathrm{Fe}$ and $\mathrm{Mg}$. The other metals are much less abundant nevertheless relatively in use as for instance $\mathrm{Ti}, \mathrm{Cu}, \mathrm{Ni}, \mathrm{Cr}$, as well as the precious metals. The abundance of elements on earth does not reflect the evolution of their discovery nor their use throughout history. For example, even if very abundant, $\mathrm{Al}$ is one of the last industrial element ever discovered: in 1825 , whereas $\mathrm{Au}, \mathrm{Ag}, \mathrm{Cu}$, and $\mathrm{Pb}$ were discovered in ancient times. Apart from gold and silver, metals have to be extracted from their ores, which are usually oxides or have been converted to the oxide. In the ore the metal exists as positive ions: $\mathrm{Fe}^{2+}$ in $\mathrm{Fe}_{2} \mathrm{O}_{3}, \mathrm{Al}^{3+}$ in $\mathrm{Al}_{2} \mathrm{O}_{3}$, $\mathrm{Na}^{+}$in $\mathrm{NaCl}$. To make the metal, the extraction involves the reduction of the metal ion by adding electrons. If a metal is reactive, it forms ions easily, i.e. oxidizes easily, therefore conversion of its ions to the free metal is difficult. The stability of the metal oxide is represented by the value of the standard driving force difference, $\Delta G^{0}$, for the formation of the oxide as $\mathrm{M}+\mathrm{O}_{2}=\mathrm{MO}_{2}$. The larger the absolute value of $\Delta G^{0}$, the larger the stability of the oxide and more difficult is the reduction of the native oxide into elemental metal.

Figure 1 shows schematically that the time at which the metals were discovered and started to be used in human advancement reflects not the abundance of the metal on the earth crust, rather the affinity of the metals to oxidize. The materials of prehistory (before $10000 \mathrm{BCE}$, the stone age) were natural ceramics, naturals polymers and composites. Weapons - always the peak of technology — were made of wood and flint, buildings and bridges of stone and wood. Naturally occurring gold and silver played a minor role in technology. The discovery of copper and bronze and then iron (the bronze age $4000 \mathrm{BCE}-1000 \mathrm{BCE}$ and the Iron age, 100 BCE-CE 1620) stimulated enormous advantages. Cast iron technology (1620s) established the dominance of metals in engineering; the evolution of steel (1850 onwards), light alloys (1940s) and special alloys since then consolidated their position (Ashby 1999). By the 1960s 'engineering materials' meant 'metals'. By that time we have learned that reactive metals such as aluminium, and all of the alkali and alkaline earth metals have to be extracted using electrolysis to reduce the ions. Less reactive metals such as $\mathrm{Zn}$ and $\mathrm{Fe}$ can be extracted using carbon or carbon monoxide to reduce the ores. Electrolysis uses very large amounts of electricity, and the process is much more expensive than reductions using $\mathrm{C}$ or $\mathrm{CO}$, thus aluminium, the commonest metal in the Earth's crust, is expensive, whereas iron is rather cheap. In recent years, research on metallic materials has been focused on advanced alloys for example on Ni-based superalloys, and ultra-light Al alloys, as well as metallic glasses, which are several times stronger than the best industrial steel 


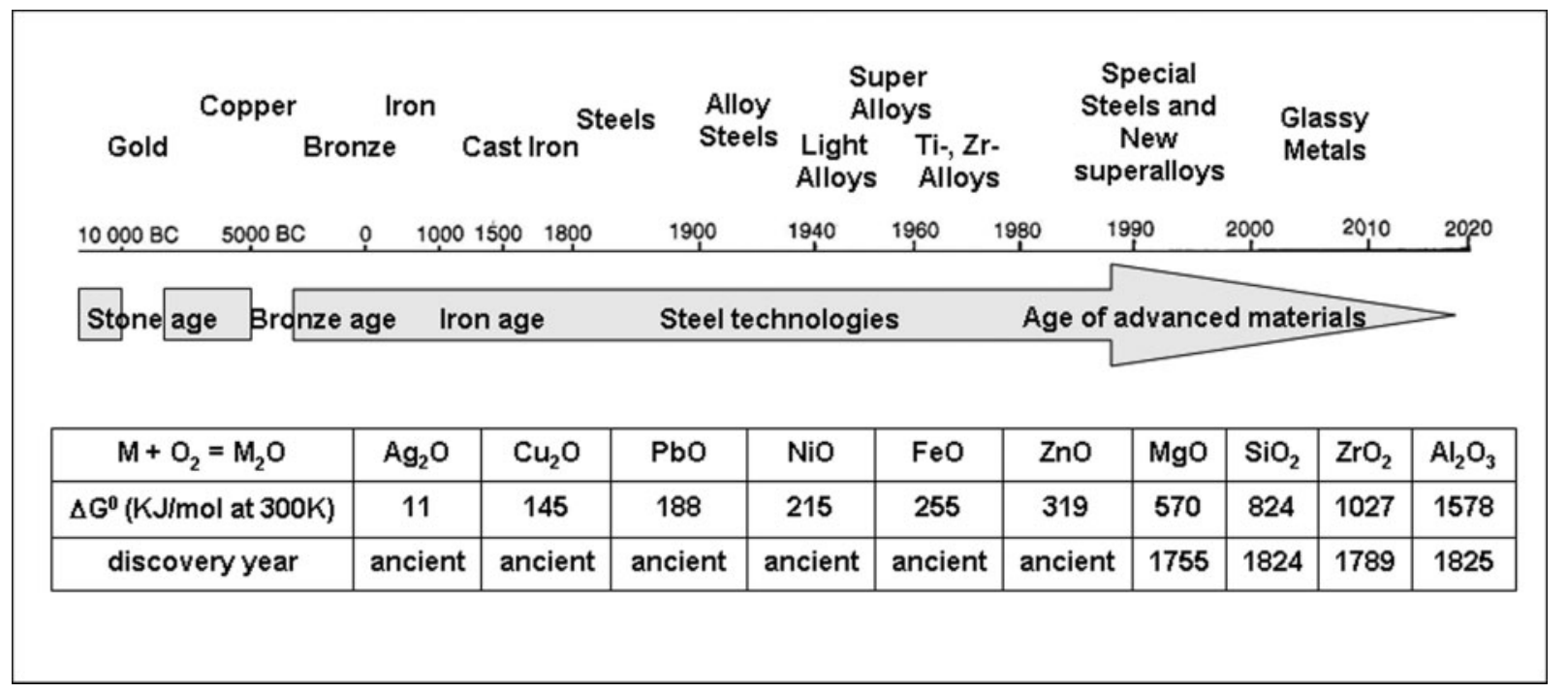

Figure 1 Schematic evolution of metals scale with correlation between the stability of selected metals oxides and the metal discovery. The scale is not linear in order to accommodate the rapid evolution of technology of the last two centuries.

and 10-times springier, therefore are promising structural materials.

\section{Metallic Glass-Forming Ability}

Up to recently, metals we always dealt with, were in the crystalline state, but recently in the late 1950s Klement, Willens \& Duwez (1960), discovered that certain alloy compositions could be quenchable into the glassy state from the liquid. It is this liquid-like structure that lacks crystalline grains as well as dislocations, which promote plastic deformation, that makes BMGs so promising. When compared to polymers or ceramics, as in Figure 2, the critical cooling rates of these first metallic glasses — or 'conventional' metallic glasses - required high cooling rates on the order of $10^{6} \mathrm{~K} \mathrm{~s}^{-1}$ and were consequently restricted to thin sections (Greer \& Ma 2007). In recent years new families of multi-component glassforming alloys that exhibit very good glass-forming ability have been discovered, such as La-Al-Ni (Inoue et al. 1991), Zr-Ni-Al-Cu (Zhang, Inoue \& Masumoto 1991), $\mathrm{Mg}-\mathrm{Cu}-\mathrm{Y}$ (Inoue, Zhang \& Masumoto 1991), Zr-Ti$\mathrm{Cu}-\mathrm{Ni}-\mathrm{Be}$ (Peker \& Johnson 1993) and, very recently, $\mathrm{Fe}-\mathrm{Mn}-\mathrm{Cr}-\mathrm{Mo}-\mathrm{C}-\mathrm{B}$ (Ponnambalam et al. 2003). These compositions can be cast into glasses at much lower cooling rates, permitting minimum sections of $1 \mathrm{~mm}$ to $1 \mathrm{~cm}$ or even larger (Greer \& Ma 2007).

Liquids that can yield bulk metallic glasses (BMGs) are multicomponet alloys with large size mismatch and a composition close to a deep eutectic (Busch, Schroers \& Wang 2007). Among the best metallic glass formers are mixtures of early transition metals - like $\mathrm{Zr}$ and $\mathrm{Ti}$ that are large in size - with late transition metals, like $\mathrm{Ni}$ and $\mathrm{Cu}$, that are smaller, and with even smaller simple metals or metalloids like Be, Boron, or Phosphorous. Figure 3 shows a twodimensional illustration of randomly and densely packed spheres for the $\mathrm{Zr}_{41.2} \mathrm{Ti}_{13.8} \mathrm{Cu}_{12.5} \mathrm{Ni}_{10.0} \mathrm{Be}_{22.5}$ (Vitreloy 1, or V1, Tang et al. 1999). The radius of the sphere labeled

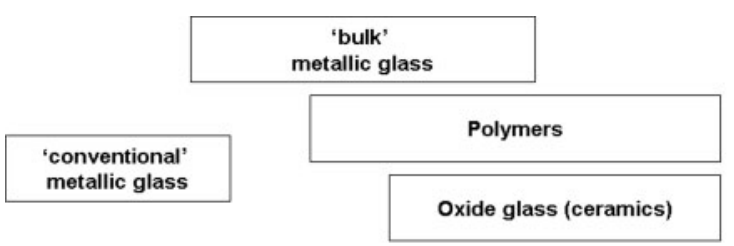

Critical cooling rate $\mathrm{K} / \mathrm{s}$

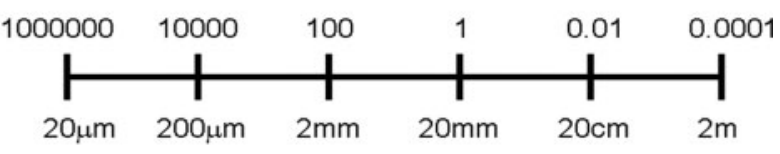

Max. thickness

Figure 2 Correlation between the critical cooling rate and the critical thickness for different classes of glass.

by a certain element is proportional to the radius of the corresponding atom. A large size mismatch like the one found in V1 destabilizes the crystalline phases and establishes a high packing density. The composition of BMGs close to a deep eutectic results in very low melting points compared to the constituent elements. Thus, the melt is thermodynamically stabilised with respect to the competing crystalline phases. Even in the metastable liquid state these bulk metallic glass formers show high thermal stability with respect to crystallization, enabling the study of the thermophysical properties of metallic melts in the supercooled state and the exploration of their properties and possible applications (Johnson 1999).

For some BMGs, this stability enables measurements of specific heat capacity (Wilde et al. 1994; Busch, Kim \& Johnson 1995), diffusion (Geyer et al. 1995; Wenwer et al. 1997; Ehmler et al. 1998), viscosity (Bakke, Busch \& Johnson 1995; Busch, Bakke \& Johnson 1998; Way, Wadhwa \& Busch 2007), local atomic motion ( $\mathrm{Li}$, Schroers \& Wu 2003) and emissivity (Busch et al. 1995) in a temperature region that was previously not accessible for 


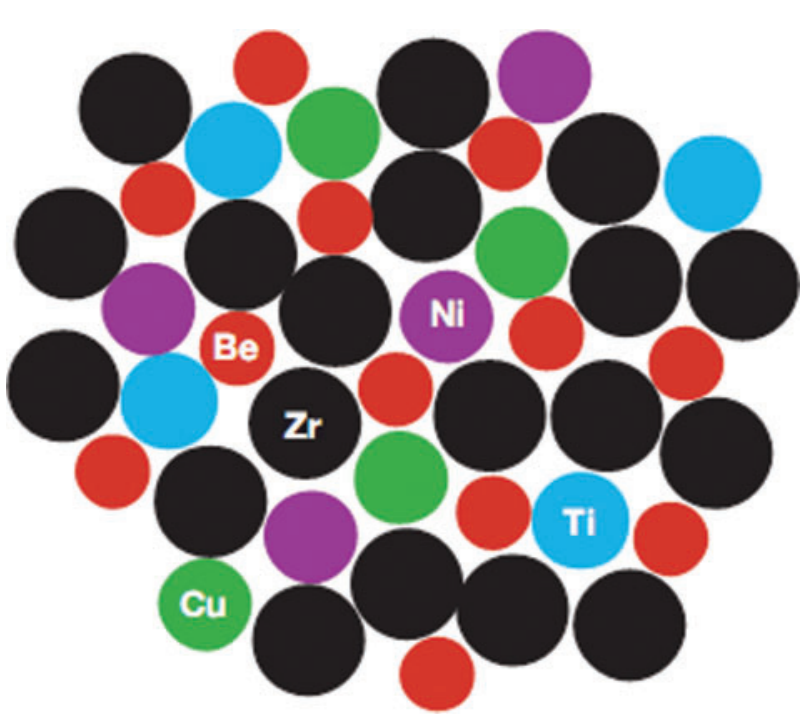

Figure 3 A two-dimensional illustration of randomly and densely packed spheres for the bulk metallic glass V1 of composition $\mathrm{Zr}_{41.2} \mathrm{Ti}_{13.8} \mathrm{Cu}_{12.5} \mathrm{Ni}_{10.0} \mathrm{Be}_{22.5}$ (Tang et al. 1999). The radius of the sphere labelled by a certain element is proportional to the radius of the corresponding atom.

the conventional metallic glasses, due to their lack of thermal stability with respect to crystallization. The stability of the supercooled liquid also enables experimental determination of the crystallization process (Kim et al. 1996), which was found to change from a nucleation-controlled mechanism at high temperatures (low undercoolings) to a growth-controlled process at low temperatures (Schroers et al. 2001). Figure 4 shows the experimentally determined time-temperature-transformation (TTT) diagram for crystallization of the supercooled liquid for V1 (Kim et al. 1996; Masuhr et al. 1999). It shows the typical C-shape or 'nose', which is the result of increasing driving force for crystallization and decreasing atomic mobility upon supercooling. The time at the nose is about $60 \mathrm{~s}$, which is amazingly long, even if compared with phase transformations within the solid state such as the austenite-pearlite transformation in steels. For previously known glassforming alloys, the times were of the order of milliseconds, resulting in the need for rapid quenching for vitrification. The TTT diagram of V1 reflects a very low critical cooling rate of about $1 \mathrm{~K} \mathrm{~s}^{-1}$, which is 5-6 orders of magnitude lower than in earlier metallic glass-forming systems.

As mentioned above, the $\mathrm{C}$-shape is the result of the competition between the increasing driving force for crystallization and the slowing of kinetics (effective diffusivity) of the atoms. In simple liquids, atomic mobility is connected to viscosity via the Stokes-Einstein relation. If steady-state nucleation is assumed, the rate of nucleation is determined by the product of a thermodynamic contribution and a kinetic contribution as

$$
I_{\mathrm{s}}=A D_{\text {eff }} \exp \left(\frac{-\Delta G^{*}}{k T}\right),
$$

where $D_{\text {eff }}$ is the effective diffusivity, $T$ is temperature, $k$ is the Boltzmann constant and $A$ is a constant. For

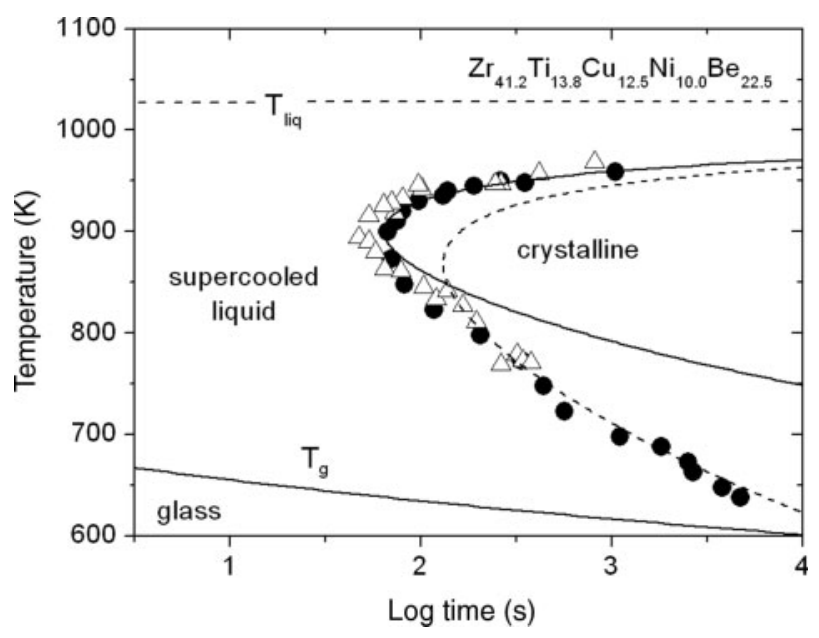

Figure 4 Time-temperature-transformation diagram for the primary crystallization of V1 (Busch et al. 2007). The data correspond to measurements by electrostatic levitation (open triangle) and processed in high-purity carbon crucibles (bold circle). The curves are the calculated times for a crystalline volume fraction of $x=10^{-4}$ using $D_{\text {eff }} \propto \eta^{-1}$ (solid curve) and using $D_{\text {eff }} \propto \exp \left(-Q_{\text {eff }} / k T\right.$ ) (dashed curve). The lines labeled $T_{\text {liq }}$ and $T_{\mathrm{g}}$ indicate the liquidus temperature and the glass transition temperature, respectively.

high temperatures, the diffusivity is set proportional to the inverse of the viscosity as $D_{\text {eff }} \propto \eta^{-1}$. The activation barrier for nucleation, $\Delta G^{*}$, is given as $\Delta G^{*}=16 \pi \sigma^{3} \Delta G^{-2}$, where $\sigma$ is the interfacial energy between liquid and solid, and $\Delta G$ is the driving force for crystallization. For simplification purposes, classical nucleation theory can be applied, resulting in

$$
t_{\mathrm{x}}=\left(\frac{3 x}{\pi I_{\mathrm{s}} u^{3}}\right)^{1 / 4}
$$

as an expression for the time to crystallize a small volume fraction, $x$. In this equation, $I_{\mathrm{s}}$ is the steady-state nucleation rate (Equation 1), and $u$ is the growth rate (Masuhr et al. 1999). If the effective diffusivity is assumed proportional to the inverse viscosity, then the crystallization time of Equation 2 is directly proportional to the viscosity which means that melts with high viscosities have a nose of the TTT diagram for crystallization to longer times and thus enhance the glass forming ability.

From these considerations, it is clear that the driving force and the viscosity, respectively, are crucial parameters for understanding the glass-forming ability of supercooled BMG-forming liquids. Viscosities of amorphous alloys have been first measured, by e.g. Chen \& Turnbull (1968); Tsao \& Spaepen (1985); Volker \& Spaepen (1989). The viscosities were determined in the glass-transition region, but crystallization did not allow measurements of the equilibrium viscosity below $10^{9} \mathrm{~Pa}$ s or for times long enough to eliminate relaxation effects. Viscosity can be now measured in bulk glass-forming systems in a much larger temperature and time range than before. The increasing viscosity on cooling reflects the decreasing atomic mobility observed in all supercooled liquids, whether they are metallic or nonmetallic. Figure 5 shows a 'fragility plot' 


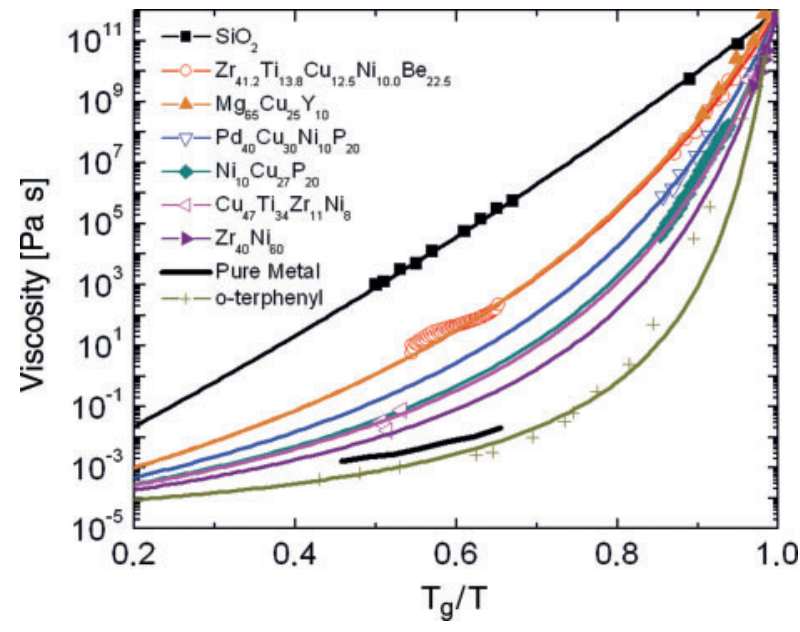

Figure 5 Fragility plot comparing the viscosities of different types of glass-forming liquids. Data on non-metallic liquids are taken from Angell (1995). Data on metallic liquids from (Bakke et al. 1995; Busch et al. 1998, 2007; Way et al. 2007).

in the form proposed by Angell (1995) in which the viscosities of different glass-forming liquids are compared in an Arrhenius plot, for which the inverse temperature axis is normalised with respect to glass-transition temperature $T_{\mathrm{g}}$. All the curves meet at $10^{12} \mathrm{~Pa} \mathrm{~s}$, corresponding to the viscosity at $T_{\mathrm{g}}$. Two extreme cases are distinguished: kinetically 'strong' liquids such as $\mathrm{SiO}_{2}$ show near-Arrhenius behaviour (straight line) and have high viscosities; on the other hand, kinetically 'fragile' liquids such as o-terphenyl exhibit a dramatic temperaturedependence of viscosity just above $\mathrm{Tg}$ and viscosities that are up to eight orders of magnitude lower than those of the strongest liquids (Angell 1995). The term 'fragile' refers to this drop in viscosity with increasing temperature and does not indicate a mechanical behavior. All pure metals (Iida \& Guthrie 1988), most metallic alloys and water have liquid viscosities at the melting point of about $10^{-3} \mathrm{~Pa}$ : they are kinetically very fragile. The equilibrium-viscosity data measured in the supercooled liquid can be described well with the Vogel-Fulcher-Tammann (VFT) relation:

$$
\eta=\eta_{0} \exp \left(\frac{D^{*} T_{0}}{T-T_{0}}\right) .
$$

Equation 3 represents a formulation of the VFT equation according to Angell (1995) in which $\eta$ is the viscosity and the pre-exponential factor $\eta_{0}$ is the viscosity at infinite temperature. The VFT equation includes the parameter $D^{*}$, which is a measure of the kinetic fragility of the liquid, and the VFT temperature $T_{0}$, where the barriers with respect to flow would go to infinity. For the most fragile liquids, $D^{*}$ is of the order of two and reaches 100 for the strongest glass-former, $\mathrm{SiO}_{2}$. An interesting feature of the fragility plot is that the viscosity curves for all liquids would meet at infinite temperature. Therefore the pre-exponential factor $\eta_{0}$ can be kept constant for all materials, and reliable fits are possible even if data are available only in the vicinity of $T_{\mathrm{g}}$. In Figure 5 the melting point is at about 0.6 on the $x$-scale. The strongest among the selected metallic-glass-forming alloys have viscosities more than four orders of magnitude higher than the pure metals. The strong liquid behaviour is the single most important contribution to the high GFA of BMG-forming alloys, because when the kinetics stays sluggish in the supercooled liquid region it drastically retards crystal nucleation and especially growth kinetics (Busch 2000; Shadowspeaker \& Busch 2004; Mukherjee et al. 2005). The high melt viscosities in multi-component BMG-forming liquids, as well as their observed small entropy differences between liquid and solid, have structural origin.

To summarize, bulk metallic glass forming liquids with high glass forming ability are multi-component eutectic alloys with large atomic size mismatch. This leads to high packing densities and small free volume, to the fact that the melt is energetically close to the crystal and most importantly that the kinetics in the liquid is slow, which means that the liquid has high viscosity. These properties are linked and kinetically suppress the crystallization.

\section{Brief Summary of Properties and Application of Bulk Metallic Glasses}

Combining some of the desirable properties of conventional crystalline metals and the formability of conventional oxide glasses and plastics, metallic glasses are highly attractive, particularly in applications in microelectromechanical systems (MEMS). The absence of grain boundaries in glassy alloys contributes to unique combinations of magnetic, electrical, chemical, and mechanical properties. The lack of dislocation defects - typical in crystalline alloys - gives metallic glasses very high strength at room-temperature, much closer to the theoretical strength than their crystalline counterparts. For example, V1 has a yield strength in tension of $1.9 \mathrm{GPa}$. They also have high elastic limit, roughly $2 \%$ elastic strain limit in tension and compression (Johnson 1999). This makes metallic glass the premier 'spring' material. By exploiting the viscous flow in the supercooled-liquid regime, near-net-shape processing can now be realized. Cast glassy alloys exhibit a shiny finish and maintain dimensional accuracy, avoiding shrinkage associated with crystallization.

\section{Conclusion}

Metallic glasses offer the large range of properties, including high strength, high elasticity, extraordinary hardness, superior resistance to wear and corrosion, and the ability to be molded on a very fine scale based on processing in the supercooled-liquid regime. Better glass forming ability and inexpensive compositions based on engineering metals such as $\mathrm{Cu}, \mathrm{Ni}, \mathrm{Fe}, \mathrm{Mg}$ and $\mathrm{Al}$ are still being sought after (Greer \& Ma 2007). This effort should continue to be encouraged, especially because a broader impact of BMGs on engineering requires simpler mixtures of elements to lower the cost and facilitate processing, recycling, and commercialization. 


\section{References}

Angell, C. A., 1995, Sci, 267, 1924

Asbhy, M. F., 1999, Materials Selection in Mechanical Design (2nd ed.; Butterworth Heinemann)

Bakke, E., Busch, R. \& Johnson, W. L., 1995, ApPhL, 67, 3260

Busch, R., Schroers, J. \& Wang, W. H., 2007, MRSBu, 32, 620

Busch, R., Kim, Y. J. \& Johnson, W. L., 1995, JAP, 77, 4039

Busch, R., Kim, Y. J., Johnson, W. L., Rulison, A. J., Rhim, W. K. \& Isheim, D., 1995, ApPhL, 66, 3111

Busch, R., Bakke, E. \& Johnson, W. L., 1998, AcMat, 46, 4725

Busch, R., 2000, JOM, 52, 39

Chen, H. S. \& Turnbull, D., 1968, JChPh, 48, 2560

Ehmler, H., Heesemann, A., Rätzke, K., Faupel, F. \& Geyer, U., 1998, PhRvL, 80, 4919

Geyer, U., Schneider, S., Johnson, W. L., Qiu, Y., Tombrello, T. A. \& Macht, M. P., 1995, PhRvL, 75, 2364

Greer, A. L. \& Ma, E., 2007, MRSBu, 32, 611

Iida, T. \& Guthrie, R. I. L., 1988, The Physical Properties of Liquid Metals (Oxford: Claredon)

Inoue, A., Zhang, T. \& Masumoto, T., 1991, MatTr, 31, 425

Inoue, A., Kato, A., Zhang, T. \& Masumoto, T., 1991, MatTr, 32, 609

Johnson, W. L., 1999, MRSBu, 24, 42
Kim, Y. J., Busch, R., Johnson, W. L., Rulison, A. J. \& Rhim, W. K., 1996, ApPhL, 68, 1057

Klement, W., Willens, R. \& Duwez, P., 1960, Natur, 187, 869

Li, L. L., Schroers, J. \& Wu, Y., 2003, PhRvL, 91, 265502

Masuhr, A., Waniuk, T. A., Busch, R. \& Johnson, W. L., 1999, PhRvL, 82, 2290

Mukherjee, S., Schroers, J., Rhim, W. K. \& Johnson, W. L., 2005, PhRvL, 92, 245501

Peker, A. \& Johnson, W. L., 1993, ApPhL, 63, 2342

Ponnambalam, V., Poon, S. J., Shiflet, G. J., Keppens, V. M., Taylor, R. \& Petculescu, G., 2003, ApPhL, 83, 1131

Schroers, J., Wu, Y., Busch, R. \& Johnson, W. L., 2001, AcMat, 49, 2773

Shadowspeaker, L. \& Busch, R., 2004, ApPhL, 85, 2508

Tang, X.-P., Geyer, U., Busch, R., Johnson, W. L. \& Wu, Y., 1999, Natur, 402, 11

Tsao, S. S. \& Spaepen, F., 1985, AcMet, 33, 1355

Volkert, C. A. \& Spaepen, F., 1989 AcMet, 37, 1355

Way, C., Wadhwa, P. \& Busch, R., 2007, AcMat, 55, 2977

Wenwer, F., Knorr, K., Macht, M. P. \& Mehrer, H., 1997, Defect and Diffusion Forum, 143-147, 831

Wilde, G., Görler, G. P., Willnecker, R. \& Dietz, G., 1994, ApPhL, 65,397

Zhang, T., Inoue, A. \& Masumoto, T., 1991, MatTr, 32, 1005 